\title{
Symmetric Nonrigid Image Registration: Application to Average Brain Templates Construction
}

\author{
Vincent Noblet $^{1}$, Christian Heinrich ${ }^{1}$, Fabrice Heitz ${ }^{1}$, \\ and Jean-Paul Armspach ${ }^{2}$ \\ ${ }^{1}$ Laboratoire des Sciences de l'Image, de l'Informatique et de la Télédétection, \\ LSIIT, UMR CNRS-ULP 7005, Bd Sébastien Brant, 67412 Illkirch Cedex, France \\ \{noblet, heinrich, heitz\}@lsiit.u-strasbg.fr \\ ${ }^{2}$ Laboratoire d'Imagerie et de Neurosciences Cognitives, \\ LINC, UMR CNRS-ULP 7191, 4 Rue Kirschleger, 67085 Strasbourg Cedex, France \\ armspach@linc.u-strasbg.fr
}

\begin{abstract}
Image registration aims at estimating a consistent mapping between two images. Common techniques consist in choosing arbitrarily one image as a reference image and the other one as a floating image, thus leading to the estimation of inconsistent mappings. We present a symmetric formulation of the registration problem that maps the two images in a common coordinate system halfway between them. This framework has been considered to devise an efficient strategy for mapping a large set of images in a common coordinate system. Some results are presented in the context of 3-D nonrigid brain MR image registration for the construction of average brain templates.
\end{abstract}

\section{Introduction}

Deformable - inter-subject - registration of 3-D medical images has received considerable attention during the last decade, as a key step for the construction and use of probabilistic anatomical atlases. The goal of nonrigid image registration is to estimate the large and highly nonlinear deformations corresponding to anatomical variability between individuals.

Common techniques consist in choosing arbitrarily one image as a reference image and the other one as a floating image, thus leading to the estimation of inconsistent mappings, i.e., the estimated transformation from the registration of $\mathrm{A}$ onto $\mathrm{B}$ may not be equal to the inverse of the transformation estimated from the registration of $\mathrm{B}$ onto $\mathrm{A}$. Some other methods tackle this problem by estimating jointly the direct transformation $T_{A B}$ (from image $A$ to image $B$ ) and the reverse transformation $T_{B A}$ (from image $B$ to image $A$ ), while minimizing some inverse consistency constraints that enforce $T_{A B}$ and $T_{B A}$ to be inverse of each other [1. Another approach consists in warping simultaneously both images while enforcing the final solution to be "halfway" from the initial images according some geodesic distance defined on the space of diffeomorphisms [2]. 
All these approaches require to estimate twice as many parameters as a standard asymmetric approach, since they involve two deformation fields.

In this paper, we present an alternative to the method presented in [2]. In the proposed framework, both images are also warped simultaneously while enforcing the final solution to be "halfway" between the initial images, but according to the Euclidean distance, similarily as in 3 . Contrary to the other existing symmetric approaches, this formulation does not require to increase the number of parameters as compared to the standard asymmetric formulation. The proposed framework is not dependent on the deformation model considered, the only assumption being the ability to enforce the deformation field and its opposite to be one-to-one mappings, and consequently to be both invertible. In this paper, we consider the framework presented in [4, that enables to enforce a B-spline deformation model to preserve topology.

The proposed framework has been extended to devise an efficient strategy for mapping a large set of images in a common coordinate system. One application is the construction of the average shape representation of a set of images. Several methods have also been proposed to this end and can be divided into two categories. The first category is based on pairwise registration. These approaches rely on the following general scheme: (i) register all the images on an arbitrarily chosen reference image, (ii) compute the mean of all estimated deformation fields and (iii) apply the inverse of the mean transformation to the reference image [5] or to the average intensity image computed from all registered images [6]. However, both approaches consider an arithmetic averaging of the displacement fields, which does not warrant the output mean transformation to have the same properties as the individual transformations, such as for example topology preservation. This is why a geodesic estimation of the mean transformation in the manifold of diffeomorphisms has been proposed in [7]. The second category gathers groupwise registration methods. These methods consist in estimating jointly the mapping associated to each image that minimize some groupwise similarity metric defined for a set of images. The optimization procedure is generally achieved while constraining the mappings to have minimum length in the sense of the Euclidean distance 8] or of some geodesic distance defined on the manifold of diffeomorphisms 9. Such approaches are very memory consuming because all images and transformation fields have to be considered simultaneously. The proposed approach can be classified in the first category since it relies on pairwise registration. The main contribution of the proposed framework is to enforce the sum of all estimated displacement fields to vanish, in order to ensure that the resulting template image is at the geometric center of the population.

This paper is organized as follows. In section 2 we present the symmetric formulation of the registration problem. In section 3. we detail how the proposed framework can be used for groupwise registration. Results on the construction of average brain templates are presented in section 4. Finally, conclusion and future prospects are given. 


\section{Symmetric Pairwise Registration}

Let us consider two images $I_{1}$ and $I_{2}$ and a transformation field $\mathbf{h}=\mathbf{I d}+\mathbf{u}$, where $\mathbf{u}$ designates the associated displacement field. The registration problem is generally stated in the following asymmetric way:

$$
\widehat{\mathbf{h}}=\arg \min _{\mathbf{h}} E_{\text {sim }}\left(I_{1}, I_{2}(\mathbf{h})\right)+E_{\text {reg }}(\mathbf{h}),
$$

where $E_{\text {sim }}$ is some function quantifying the similarity between $I_{1}$ and $I_{2}(\mathbf{h})$ and $E_{\text {reg }}$ a regularization term that penalizes transformations that are not smooth. To enforce symmetry in the formulation of the registration problem, we propose to consider the following optimization problem:

$$
\widehat{\mathbf{u}}=\arg \min _{\mathbf{u}} E_{\text {sim }}\left(I_{1}(\mathbf{I d}-\mathbf{u}), I_{2}(\mathbf{I d}+\mathbf{u})\right)+E_{r e g}(\mathbf{u}),
$$

subject to the constraint that "Id $-\mathbf{u}$ and $\mathbf{I d}+\mathbf{u}$ preserve topology". Considering a displacement field and its opposite ensures that each point in the common coordinate system is "halfway" from the corresponding points in the two images according to the Euclidean distance (see Fig. 1). To obtain the transformation between the two images, the inverse of one of the transformations has to be computed and composed with the other transformation.

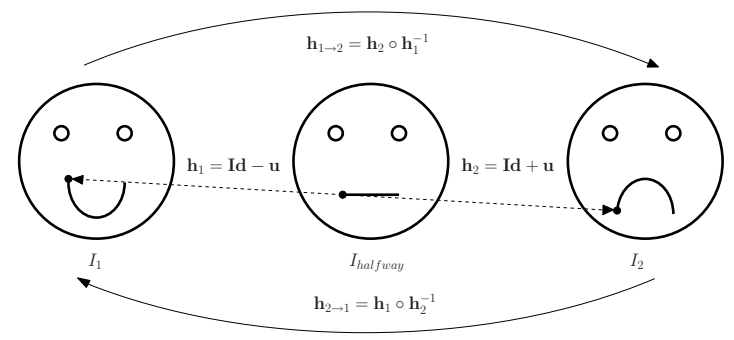

Fig. 1. The proposed symmetric formulation

The proposed framework is not dependent on the deformation model considered, the only assumption being the ability to enforce the deformation field and its opposite to be one-to-one mappings, and consequently to be both invertible. Here, we consider the framework presented in [4] that enables to enforce a Bspline deformation model to preserve the topology. The application presented in this paper is single-modal inter-subject brain image registration. We consider the sum of squared differences as similarity criterion 1$]$ and the elastic membrane

\footnotetext{
${ }^{1}$ Using the sum of squared differences generally requires an intensity normalization scheme so that the intensities are comparable in both images [10. We do not use the method presented in [10 since the two images do not play a symmetric role in this method. This is why a simple linear scaling of the intensities considering mean and standard deviation is considered here. Further work will concern the symmetrization of the intensity normalization method presented in [10, which has shown to significantly improve the registration quality in the context of single-modal brain image registration.
} 


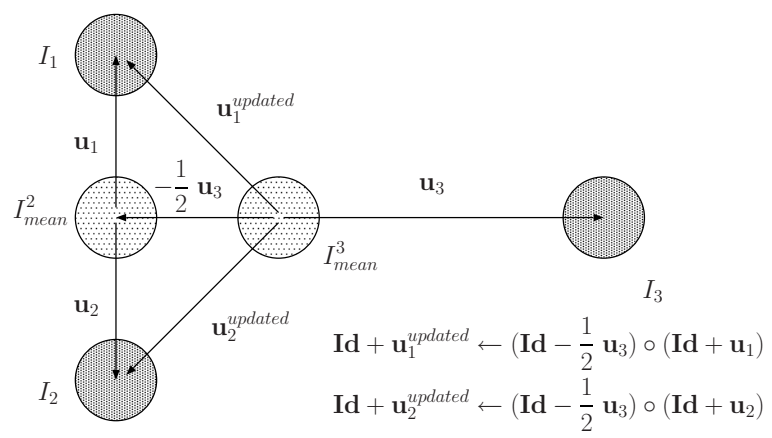

Fig. 2. Scheme illustrating the proposed strategy for building the average template. First, $I_{\text {mean }}^{2}$ is obtained from the pairwise symmetric registration of $I_{1}$ and $I_{2} . I_{\text {mean }}^{3}$ is then obtained by considering the registration of $I_{\text {mean }}^{2}$ and $I_{3}$.

energy as regularization term. Estimation of the parameters is achieved using the Lenvenberg-Marquardt algorithm and the constraints on $\mathbf{I d}-\mathbf{u}$ and $\mathbf{I d}+\mathbf{u}$ to preserve the topology are enforced using the optimization scheme described in 4. Inversions of deformation fields are achieved using the algorithm presented in [11, which enables to hold the maximal inversion error below some user-defined accuracy. We consider a maximal inversion error of 0.01 in our experiments.

\section{Groupwise Registration}

In this section, we extend the method to the registration of $N$ images. The goal is the following: given $N$ images $I_{1}, \ldots, I_{N}$, estimate $N$ deformation fields $\mathbf{u}_{1}, \ldots, \mathbf{u}_{N}$ so that the $\mathrm{N}$ warped images $I_{1}\left(\mathbf{I d}+\mathbf{u}_{1}\right), \ldots, I_{N}\left(\mathbf{I d}+\mathbf{u}_{N}\right)$ are as close as possible of each other, while constraining the sum of all deformation fields to vanish, i.e., :

$$
\forall \mathbf{s} \in \Omega, \sum_{i=1}^{N} \mathbf{u}_{i}(\mathbf{s})=\mathbf{0},
$$

where $\Omega$ is the overlapping area of all warped image supports. Solving this constrained optimization problem by estimating jointly the $N$ deformation fields is highly demanding in memory and computing power. A similar problem has already been tackled by Studholme et al [8, but instead of considering the hard constraint (3), they considered an additional penalty term in their objective function. Here, we propose a framework enforcing constraint (3) by considering the following theorem related to centroïd computation:

Theorem 1. Given a set of $i+1$ points $\left\{P_{1}, \ldots, P_{i+1}\right\}$ and assuming that $A$ is the centroïd of $\left\{P_{1}, \ldots, P_{i}\right\}$, then the centrö̈d $G$ of $\left\{P_{1}, \ldots, P_{i+1}\right\}$ verifies:

$$
i \overrightarrow{G A}+\overrightarrow{G P_{i+1}}=\overrightarrow{0}
$$




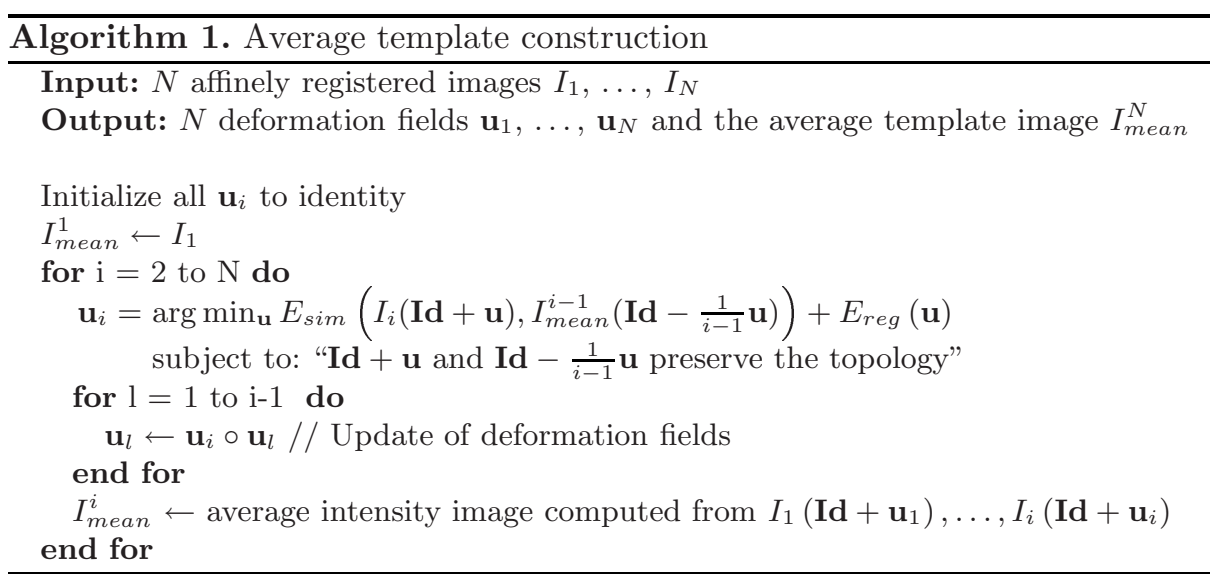

Let $I_{\text {mean }}^{i}$ be the average intensity image 2 computed from a set of $i$ registered images $I_{1}\left(\mathbf{h}_{1}\right), \ldots, I_{i}\left(\mathbf{h}_{i}\right)$, the corresponding deformation fields $\mathbf{u}_{1}, \ldots, \mathbf{u}_{i}$ verifying condition (3). To estimate the average intensity image $I_{\text {mean }}^{i+1}$ when considering an additional image $I_{i+1}$, the following pairwise registration between $I_{\text {mean }}^{i}$ and $I_{i+1}$ has to be estimated:

$$
\widehat{\mathbf{u}}_{i+1}=\arg \min _{\mathbf{u}} E_{\text {sim }}\left(I_{\text {mean }}^{i}\left(\mathbf{I d}-\frac{1}{i} \mathbf{u}\right), I_{i+1}(\mathbf{I d}+\mathbf{u})\right)+E_{\text {reg }}(\mathbf{u}) .
$$

All previously estimated transformations $\mathbf{h}_{1}, \ldots, \mathbf{h}_{i}$ are then updated by composing them with the estimated transformation $\mathbf{I d}-\frac{1}{i} \widehat{\mathbf{u}}_{i+1}$. Using this strategy ensures that the deformation fields $\mathbf{u}_{1}, \ldots, \mathbf{u}_{i+1}$ fulfill also condition (3). Figure2 illustrates this basic idea. The complete algorithm for building the average template is detailed in Alg. [1]

\section{Results: Application to Average Brain Template Construction}

We compare the proposed method for average template construction, denoted in the sequel Method1, with a similar approach to those presented in [56], denoted in the sequel Method2, which consists in the following 5 steps: (i) register all images on an arbitrarily chosen reference image using the symmetric registration method presented in section 2 (Eq. 2), (ii) compute the average intensity image, (iii) compute the mean transformation field, (iv) estimate the inverse of the mean transformation field using the inversion algorithm proposed in 11 and $(\mathrm{v})$ warp the average intensity image using the inverse mean transformation field. Comparisons have been carried out on a database of 15 MR T1-weighted brain images (matrix size $=256^{3}$ ).

\footnotetext{
${ }^{2}$ Notice that in our implementation, the intensities of all images are firstly corrected using mean and standard deviation normalization using the first image $I_{1}\left(\mathbf{h}_{1}\right)$ as reference image before computing the average intensity image.
} 

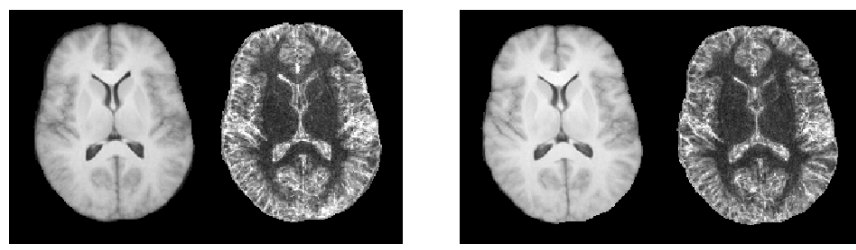

Fig. 3. Average intensity image and corresponding standard deviation image obtained with Method1 (left) and with Method2 (right)

We consider four indices to evaluate and compare the two approaches. The means and standard deviations of all these indices, computed over the overlapping area of all warped image supports, are reported in Table 1 . The first index evaluates if condition (3) is well fulfilled by considering the norm of the sum of all displacement fields:

$$
S D F(\mathbf{s})=\left\|\sum_{i=1}^{N} \mathbf{u}_{i}(\mathbf{s})\right\|
$$

In Table 1, we can see that condition (3) is fulfilled 3 by Method1 whereas Method2 yields a mean $S D F$ index nearly equal to one voxel. The second index considered aims at evaluating if the estimated average template is really at the geometric center of the population by considering the mean Euclidean distance from the average template:

$$
\operatorname{MDT}(\mathbf{s})=\frac{1}{N} \sum_{i=1}^{N}\left\|\mathbf{u}_{i}(\mathbf{s})\right\| .
$$

A lower mean MDT index value has been obtained for Method1, highlighting that the average template estimated with this method is closer to the centroïd of the population than the one estimated with Method2. Then, we investigate the sensitivity of the proposed approach with respect to the order in which the images are processed. Thus, we ran Method1 twice considering two sequences differing in the order in which images were processed, thus leading to the estimation of two sets of deformation fields denoted $\left\{\mathbf{u}_{i}^{A}\right\}_{i=1 \cdots N}$ and $\left\{\mathbf{u}_{i}^{B}\right\}_{i=1 \cdots N}$. In a similar way, we ran Method2 twice considering two different reference images, thus also leading to two sets of deformation field 4 . Then, we consider the Euclidean distance between the two templates estimated in experiments $A$ and $B$ :

$$
\operatorname{DBT}(\mathbf{s})=\frac{1}{2 N} \sum_{i=1}^{N}\left\|\mathbf{h}_{i}^{A}(\mathbf{s}) \circ \mathbf{h}_{i}^{B}(\mathbf{s})^{-1}-\mathbf{I d}\right\|+\left\|\mathbf{h}_{i}^{B}(\mathbf{s}) \circ \mathbf{h}_{i}^{A}(\mathbf{s})^{-1}-\mathbf{I d}\right\| .
$$

\footnotetext{
${ }^{3}$ The mean of the $S D F$ index is not exactly equal to zero for Method1 because of some residual errors introduced by the inversion scheme and by the procedure for composing transformation fields.

${ }^{4}$ For Method2, the deformation field $\mathbf{u}_{i}$ is obtained by composing the transformation estimated from registration of $I_{i}$ onto the reference image with the inverse of the mean transformation field.
} 
Table 1. Results of the comparison of Method1 with Method2

\begin{tabular}{|c|c|c|}
\hline Index & Method1 & Method2 \\
\hline$S D F$ & $1.690 .10^{-5} \pm 3.71 .10^{-6}$ & $0.9394 \pm 0.556$ \\
\hline$M D T$ & $1.416 \pm 0.295$ & $1.607 \pm 0.447$ \\
\hline$D B T$ & $0.615 \pm 0.239$ & $2.288 \pm 0.737$ \\
\hline$V I$ & $1091 \pm 483$ & $1068 \pm 483$ \\
\hline
\end{tabular}

As expected, the mean of this index is lower for Method1 than for Method2, thus highlighting the ability of the proposed approach to reduce the bias in the estimation of the average template. Some perspectives are given in the next section to further reduce the residual bias still persisting with Method1. These three indices are not sufficient to evaluate the performance of a method for average template construction, since best results can be obtained by considering vanishing displacement fields. This is why we have also to evaluate the quality of the estimated average intensity image. We can see on Fig. 3 that the two methods lead to quite similar average intensity images, which are not so much blurred, thus highlighting the ability of both approaches to cope with the high interindividual brain anatomical variability. We also consider the variance image (Fig. 3):

$$
V I(\mathbf{s})=\sqrt{\frac{1}{N} \sum_{i=1}^{N}\left\|I_{i}\left(\mathbf{s}+\mathbf{u}_{i}(\mathbf{s})\right)-I_{\text {mean }}(\mathbf{s})\right\|^{2}} .
$$

We can see that Method1 and Method2 lead also to quite similar variances, the variance obtained with Method1 being slightly higher. This observation is consistent with the fact that Method1 is more constrained than Method2 because of condition (3), thus preventing to make at best decrease the dissimilarities between all images. The perspectives given in the next section relying on the use of groupwise similarity distance may lead to some improvements of the proposed method on this point. Finally, it has also to be noticed that the two methods are quite equivalent in term of computational burden and memory requirement since they both rely on pairwise registration. The only overhead introduced in Method1 compared to Method2 is the update of the deformation fields and the computation of the average intensity image that have to be done at each iteration.

\section{Conclusion and Future Prospects}

We have presented a symmetric formulation for nonrigid image registration that has been extended to devise an efficient strategy for average template construction. The main advantage of the proposed method is that it can easily enforce the sum of all deformation fields to vanish, while still being neither too much time consuming nor memory demanding since it relies only on pairwise registrations. The proposed framework is versatile and can also be used with some 
groupwise similarity distance. For instance, it would be possible to refine the set of transformation fields $\mathbf{h}_{1}, \ldots, \mathbf{h}_{N}$ estimated with the proposed method by considering a groupwise similarity distance $E_{g w}$. This may be done by considering the following optimization problem for each image $I_{i}$ until convergence:

$$
\widehat{\boldsymbol{\Delta}}_{i}=\arg \min _{\boldsymbol{\Delta} \mathbf{u}} E_{g w}\left[I_{1}\left(\left(\mathbf{I d}-\frac{\mathbf{\Delta u}}{N-1}\right) \circ \mathbf{h}_{1}\right), \cdots, I_{i}\left((\mathbf{I d}+\boldsymbol{\Delta} \mathbf{u}) \circ \mathbf{h}_{i}\right), \cdots, I_{N}\left(\left(\mathbf{I d}-\frac{\Delta \mathbf{u}}{N-1}\right) \circ \mathbf{h}_{N}\right)\right] .
$$

Further work will be done to implement and evaluate this extension of the proposed approach.

\section{References}

1. Christensen, G., Johnson, H.: Consistent image registration. IEEE Transactions on Medical Imaging 20(7), 568-582 (2001)

2. Avants, B., Epstein, C., Grossman, M., Gee, J.: Symmetric diffeomorphic image registration with cross-correlation: Evaluating automated labeling of elderly and neurodegenerative brain. Medical Image Analysis 12(1), 26-41 (2008)

3. Studholme, C., Drapaca, C.S., Iordanova, B., Cardenas, V.: Deformation-based mapping of volume change from serial brain. MRI in the presence of local tissue contrast change 25(5), 626-639 (2006)

4. Noblet, V., Heinrich, C., Heitz, F., Armspach, J.P.: 3-D deformable image registration: a topology preservation scheme based on hierarchical deformation models and interval analysis optimization. IEEE Transactions on Image Processing 14(5), 553-566 (2005)

5. Christensen, G.E., Johnson, H.J., Vannier, M.W.: Synthesizing average 3D anatomical shapes. Neuro Image 32(1), 146-158 (2006)

6. Guimond, A., Meunier, J., Thirion, J.P.: Average brain models: a convergence study. Computer Vision and Image Understanding 77(2), 192-210 (2000)

7. Avants, B., Gee, J.: Geodesic estimation for large deformation anatomical shape averaging and interpolation. Neuro Image 23 (suppl. 1), S139-S150 (2004)

8. Studholme, C., Cardenas, V.: A template free approach to volumetric spatial normalization of brain anatomy. Pattern Recognition Letters 16(10), 1191-1202 (2004)

9. Joshi, S., Davis, B., Jomier, M., Gerig, G.: Unbiased diffeomorphic atlas construction for computational anatomy. Neuro Image 23 (suppl. 1), S151-S160 (2004)

10. Noblet, V., Heinrich, C., Heitz, F., Armspach, J.P.: Retrospective evaluation of a topology preserving non-rigid registration method. Medical Image Analysis 10(3), 366-384 (2006)

11. Noblet, V., Heinrich, C., Heitz, F., Armspach, J.P.: Accurate inversion of 3-D transformation fields. IEEE Transactions on Image Processing (accepted for publication, 2008) 